\title{
Large Eddy Simulations of the Influence of Piston Position on the Swirling Flow in a Model Two-Stroke Diesel Engine
}

\author{
A. Obeidat, T. Schnipper, K. M. Ingvorsen, S. Haider, K. E. Meyer \\ Department of Mechanical Engineering, Technical University of Denmark, \\ DK-2800 Kgs. Lyngby, Denmark \\ S. Mayer \\ MAN Diesel $\& 3$ Turbo, Teglholmsgade 41, DK-2450 Copenhagen SV, Denmark \\ J. H. Walther \\ Department of Mechanical Engineering, Technical University of Denmark, \\ DK-2800 Kgs. Lyngby, Denmark \\ Chair of Computational Science, ETH Zürich, CH-8092 Zürich, Switzerland
}

\author{
Received (Day Month Year) \\ Revised (Day Month Year)
}

Purpose- the purpose of this paper is to study the effect of piston position on the in-cylinder swirling flow in a simplified model of a large two-stroke marine diesel engine.

Design/Methodology/Approach- Large Eddy Simulations with four different models for the turbulent flow are used: a one-equation model, a dynamic one-equation model, a localized dynamic one-equation model and a mixed-scale model. Simulations are carried out for two different geometries corresponding to $100 \%$ and $50 \%$ open scavenge ports.

Findings- It is found that the mean tangential profile inside the cylinder changes qualitatively with port closure from a Lamb-Oseen vortex profile to a solid body rotation while the axial velocity changes from a wake-like profile to a jet-like profile. The numerical results are compared with particle image velocimetry measurements (?) and in general we find a good agreement.

Limitations/implications- Considering the complexity of the real engine, we designed the engine model using the simplest configuration possible. The setup contains no moving parts, the combustion is neglected and the exhaust valve is discarded.

Originality/value- Studying the flow in a simplified engine model, the setup allows studies of fundamental aspects of swirling flow in a uniform scavenged engine. Comparing the four turbulence models, the local dynamic one-equation model is found to give the best agreement with the experimental results.

Keywords: diesel engine; swirling flow; scavenge flow; large eddy simulation; particle image velocimetry

\section{Introduction}

The scavenging process in two-stroke marine diesel engines removes combustion gases from the engine cylinder and fills up the cylinder with the fresh air charge for 
the next cycle. Understanding the scavenging flow is crucial for the development of such engines, since it affects fuel consumption, engine cooling and production of pollutants (?). In this work we consider the uniflow scavenging process where the exhaust valve is located at the cylinder head and the scavenge ports are located in the cylinder liner near the bottom dead centre (BDC). When the piston moves towards BDC, the exhaust valve opens while the piston slides to uncover the scavenge ports, thus initiating the scavenge process. The scavenge ports are angled such that the flow enters with an overall swirl in the cylinder. In the succeeding compression stroke this swirling flow produces a pocket of swirling fresh air, which is important for the optimization of the fuel spray mixing in the subsequent combustion phase.

Due to the rich fluid dynamic phenomena and many applications, there is a large body of work on flows with an overall swirl. Examples include the structure of helical vortices in swirling flow in a confined "vortex chamber" at moderate Reynolds number (?), vortex breakdown (??), vortex precession, and the derived influence on combustors and dust separators (?).

Recently, ? considered the effect of piston position on the scavenging and swirling flow in a simplified model of a large two-stroke marine diesel engine cylinder. Recognizing the complexity of a real engine, ? studied the flow in a "simplest possible" engine model: it contains no moving parts, and the set-up itself as well as the boundary conditions are rotationally symmetric, the exhaust valve is discarded, and the combustion is neglected. Of notable mention is the great care that was taken to impose an axisymmetric swirl by placing 60 thin guide vanes upstream of a contraction section in the inlet. By design, the set-up allows studies of fundamental aspects of the swirling flow in a uniflow scavenged engine. The effect of piston position was elucidated from time-averaged three-dimensional velocity fields of the air flow, obtained with stereo particle image velocimetry (SPIV) in the cylinder.

The current work is devoted to numerical modeling of the in-cylinder confined turbulent swirling flow of the experiment by ?. We consider two cases of static piston position corresponding to $50 \%$ and $100 \%$ open scavenge ports, respectively. These cases yield qualitatively different regimes of the swirling flow, and complements previous studies that typically consider the case of fully open scavenge ports (?). The experimental results will be compared with simulations using four different Large Eddy Simulation (LES) models: the one-equation model (OEM) cf. ?, the dynamic one-equation model (DOEM) cf. ?, the localized dynamic one-equation model (LDOEM) cf. ?, and the mixed-scale model (MSM) cf. ?. The numerical models are implemented within the OpenFOAM platform. It is noted that swirling flows are notoriously difficult to simulate due to the inherent anisotropic turbulence. For example, Reynolds-Averaged Navier-Stokes (RANS) models are known to overestimate the rate of decay of swirl due to the eddy viscosity approach (?).

The remaining part of the paper is organised as follows. Section 2 describes the numerical method and turbulence models, Section 3 describes the computational domain and boundary conditions, and Section 4 presents the results of the compu- 
tations and compares them with measurements. Section 5 discusses the results and presents conclusions.

\section{Large eddy simulation}

In order to model the confined turbulent swirling flow in ? we make use of Large Eddy Simulations, where the flow is governed by the spatially filtered Navier-Stokes equations cf. ?

$$
\begin{aligned}
\frac{\partial \bar{u}_{j}}{\partial x_{j}} & =0 \\
\frac{\partial \bar{u}_{i}}{\partial t}+\frac{\partial \bar{u}_{i} \bar{u}_{j}}{\partial x_{j}} & =-\frac{1}{\rho} \frac{\partial \bar{p}}{\partial x_{i}}+\nu \frac{\partial^{2} \bar{u}_{i}}{\partial x_{j}^{2}}+\frac{\partial \tau_{i j}}{\partial x_{j}}, \\
\tau_{i j} & =\bar{u}_{i} \bar{u}_{j}-\bar{u}_{i} u_{j} .
\end{aligned}
$$

Here, $t$ denotes time, $\bar{u}_{i}$ the filtered velocity component in the cartesian $\left(x_{i}\right)$ coordinate directions, $\bar{p}$ the filtered pressure, $\rho$ the density, and $\nu$ is the kinematic viscosity. The resolved large scales $\left(\bar{u}_{i}, \bar{p}\right)$ are explicitly computed on the computational grid, whereas small-scales fluctuations $\left(u_{i}-\bar{u}_{i}, p-\bar{p}\right)$ are modeled through subgrid-scale stresses: $\tau_{i j}$

$$
\tau_{i j}-\frac{1}{3} \delta_{i j} \tau_{k k}=-2 \nu_{\mathrm{t}} \bar{S}_{i j}
$$

cf. ?. Here $\nu_{\mathrm{t}}$ is the subgrid eddy viscosity, $\delta_{i j}$ is the Kronecker delta, and $\bar{S}_{i j}$ is the resolved strain stress tensor defined as

$$
\bar{S}_{i j}=\frac{1}{2}\left(\frac{\partial \bar{u}_{i}}{\partial x_{j}}+\frac{\partial \bar{u}_{j}}{\partial x_{i}}\right) .
$$

The trace of the subgrid scale stress $\left(\tau_{k k}\right)$ is usually included in the pressure $(\bar{p})$.

\subsection{One-equation model}

The One-Equation model (OEM) proposed by ? is based on a transport equation for the subgrid-scale turbulent kinetic energy $(k)$

$$
\frac{\partial k}{\partial t}+\frac{\partial}{\partial x_{j}}\left(k \bar{u}_{j}\right)=\frac{\partial}{\partial x_{j}}\left[\left(\nu+\nu_{\mathrm{t}}\right) \frac{\partial k}{\partial x_{j}}\right]+2 \nu_{\mathrm{t}} \bar{S}_{i j} \bar{S}_{i j}-C_{\epsilon} \frac{k^{\frac{3}{2}}}{\Delta},
$$

where

$$
k=\frac{1}{2} \overline{u_{i} u_{i}}-\frac{1}{2} \bar{u}_{i} \bar{u}_{i},
$$

see also ????. Here, $\Delta=\left(\Delta_{x} \Delta_{y} \Delta_{z}\right)^{\frac{1}{3}}$, where $\Delta_{x}, \Delta_{y}$, and $\Delta_{z}$ denote the grid spacing in the $x, y$, and $z$ directions, respectively. The constant $C_{\epsilon}=0.93$ is obtained from Kolmogorov's $5 / 3$ law cf. (?). The eddy viscosity is modeled as

$$
\nu_{\mathrm{t}}=C k^{\frac{1}{2}} \Delta
$$

where the dimensionless parameter $C=0.094$. 


\subsection{Dynamic one-equation models}

The Dynamic One-Equation Eddy-viscosity Model (DOEM), allows the parameter $C$ to vary in space and time during the simulation. The stress tensor is modeled as

$$
\tau_{i j}-\frac{1}{3} \delta_{i j} \tau_{k k}=C \alpha_{i j},
$$

where $\alpha_{i j}$ is obtained from

$$
\alpha_{i j}=-2 \Delta^{2}\left(2 \bar{S}_{k l} \bar{S}_{k l}\right)^{\frac{1}{2}} \bar{S}_{i j}
$$

cf. (?). The basic idea of the DOEM is to employ a second explicit filter $(\sim)$ with $\widetilde{\Delta}=2 \Delta$ to the filtered Navier-Stokes equations (Eqs. (1-3)), which yields the subtest-scale (STS) stress tensor

$$
T_{i j}=\widetilde{\overline{u_{i} u_{j}}}-\tilde{\tilde{u}}_{i} \tilde{\bar{u}}_{j} .
$$

$T_{i j}$ can be modeled as

$$
T_{i j}-\frac{1}{3} \delta_{i j} T_{k k}=C \beta_{i j},
$$

where $\beta_{i j}$ is

$$
\beta_{i j}=-2 \widetilde{\Delta}^{2}\left(2 \tilde{\bar{S}}_{k l} \tilde{\bar{S}}_{k l}\right)^{\frac{1}{2}} \tilde{\bar{S}}_{i j} .
$$

The Germano identity (?) expresses the resolved turbulent stress $L_{i j}$ as

$$
L_{i j}=T_{i j}-\tau_{i j},
$$

and

$$
L_{i j}-\frac{1}{3} \delta_{i j} L_{k k}=C\left(\beta_{i j}-\alpha_{i j}\right) .
$$

An approximation for $C$ is obtained by minimizing in a least-squares sense the error

$$
Q=\left(L_{i j}-\frac{1}{3} \delta_{i j} L_{k k}+C M_{i j}\right)^{2},
$$

where $M_{i j}=\alpha_{i j}-\beta_{i j}$. The minimum error is obtained at $\frac{\partial Q}{\partial C}=0$, with

$$
C=-\frac{L_{i j} M_{i j}}{M_{k l} M_{k l}} .
$$

The $C$ obtained from Eq. (17) can attain both both positive and negative values. A negative value implies a locally negative eddy viscosity which may result in an unstable solution. To circumvent this, ? proposed to average $C$ across homogeneous directions. The standard DOEM model in OpenFOAM performs a simple global average, hence the $C$ in the present DOEM model is only allowed to vary in time. The OpenFOAM implementation of the Localized Dynamic One-Equation Eddyviscosity Model (LDOEM) proposed by ? performs a local average using a simple top-hat filter. 


\subsection{Mixed scale model}

The Mixed Scale Model (MSM) proposed by ? and ? is based on the velocityvorticity formulation of the Navier-Stokes equations. Two spatial filters are used in the simulation, a filter denoted by $\left(^{-}\right)$is used on the fine mesh, and a test filter denoted by $(\sim)$ on the coarse mesh. In Eq. (3) we defined $\tau_{i j}$ as the turbulent stresses from the spatial filtering, the turbulent stresses are modeled with an eddy viscosity as

$$
\tau_{i j}=\nu_{\mathrm{t}} 2 \bar{S}_{i j}-\frac{2}{3} k \delta_{i j}
$$

The eddy viscosity $\left(\nu_{\mathrm{t}}\right)$ is determined by the mixed-scale turbulence model

$$
\nu_{\mathrm{t}}=C\left|\bar{\omega}_{i}\right|^{\alpha} k^{(1-\alpha) / 2} \Delta^{(1+\alpha)} .
$$

Here, $\bar{\omega}_{i}=\partial_{j} \bar{u}_{k}-\partial_{k} \bar{u}_{j}$ is the vorticity, and $0 \leq \alpha \leq 1$ is a constant. The turbulent kinetic energy $(k)$ is estimated from the test filter $\left(^{\sim}\right)$ as follows

$$
k=\frac{1}{2} \sum_{i=1}^{3}\left(u_{i}-\bar{u}_{i}\right)^{2} \approx \frac{1}{2} \sum_{i=1}^{3}\left(\bar{u}_{i}-\tilde{\tilde{u}}_{i}\right)^{2},
$$

Finally, from Eq. (19) we obtain the pure vorticity model if $\alpha=1$

$$
\nu_{\mathrm{t}}=C|\bar{\omega}| \Delta^{2} .
$$

If $\alpha=0$ we obtain the Bardina model (?)

$$
\nu_{\mathrm{t}}=C k^{\frac{1}{2}} \Delta
$$

From a previous study (?) it was found that the model performs best when $\alpha=\frac{1}{2}$ such that

$$
\nu_{\mathrm{t}}=C|\bar{\omega}|^{\frac{1}{2}} k^{\frac{1}{4}} \Delta^{\frac{3}{2}}
$$

\section{Numerical set-up}

The LES models are solved using the pisoFoam solver which is an OpenFOAM (version 1.6) build-in transient solver for incompressible and turbulent flows, where velocity and pressure are coupled using the PISO scheme (?). For the simulations we use the following numerical schemes: backward second order scheme for the temporal derivative and a second order central difference scheme for terms involving gradient, divergence and Laplacian operators. Finally, we make use of linear interpolation onto the subgrid-scale.

\subsection{Computational domain}

The computational domain is sketched in Fig. 1 and consists of an inlet section, a cylinder, an exhaust pipe and an outlet. The cylinder radius is $R$ and all other 
$\mathrm{R} B \mathrm{~B} R$ inlet inlet cylinder cylinder exhaust exhaust outletoutlet pistoniston intakeintake port $\mathrm{R}$ P5 $R$ Ri1.1 $R$ Ro0.58R R $R$ Lc $L_{\mathrm{c}}=8 R \operatorname{L\oplus 5} R$ h1.1 $R$ x $x$ yy zz $0^{z_{0}} 1^{z_{1}} 2^{z_{2}} 3^{z_{3}} 4^{z_{4}} 5^{z_{5}}$

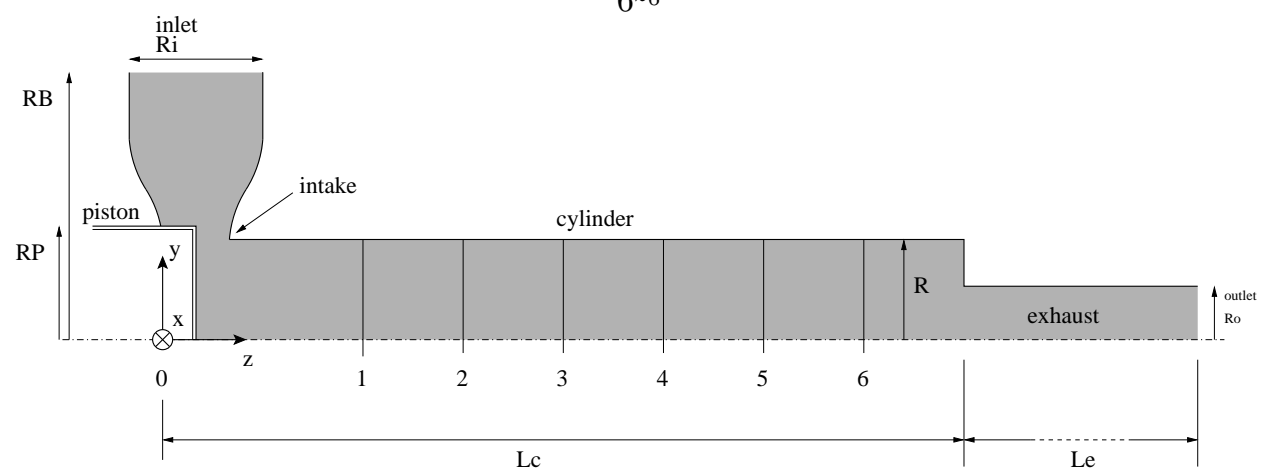

Fig. 1. Sketch of the cylindrical computational domain shown in grey shades, in the case where the piston covers the intake by $50 \%$. Flow enters the domain through the horizontal inlet section and exits the domain through the vertical outlet. Notice that the exhaust is shortened in the figure. Data are extracted at the cross-sectional planes $z_{\{0-6\}} / R=\{0.00,1.72,2.77,3.82,4.87,5.93,6.98\}$.

dimensions are shown in Fig. 1. Flow enters uniformly along a direction perpendicular to the cylinder axis, with an azimuthal velocity component, which ensures the overall in-cylinder swirling flow. The dimensions match the experiment by ?.

We simulate the flow on a grid with 8 million cells. The time step is chosen to ensure a stable and converged solution and to keep the Courant number in the range of $\bar{u} \delta t / \Delta<1$ This is satisfied with a time step $\delta t V_{\mathrm{b}} / L_{\mathrm{c}}=3.4 \cdot 10^{-4}$, where $V_{\mathrm{b}}$ is the bulk average flow speed in the cylinder and $L_{\mathrm{c}}$ is the length of the cylinder. Supplementary simulations on a 12-million node grid (not included here) show that the simulated flows are independent of the spatial discretization.

\subsection{Boundary conditions}

At the inlet of the computational domain a uniform radial and tangential velocity is prescribed such that the flow enters with constant radial speed $V_{r, \mathrm{i}}=0.23 V_{\mathrm{b}}$ and constant tangential speed $V_{\theta, \mathrm{i}}=0.11 V_{\mathrm{b}}$, which is consistent with the $26^{\circ}$ flow angle measured by ?. At the outlet we require a zero velocity gradient. On the solid walls cf. Fig. 1, a no-slip boundary condition is prescribed.

We have performed simulations with $0 \%, 5 \%$ and $10 \%$ turbulence intensity (in the form of white noise) specified at the inlet, and found no impact on the results. For all simulations presented, a zero turbulence intensity is specified at the inlet.

\section{Results}

We study the effect of piston position by simulating the flow in two different geometries. One case corresponds to $100 \%$ open intake (Section 4.1) and the other 
case corresponds to $50 \%$ open intake (Section 4.2). We discuss in detail the mean axial and tangential velocity profiles obtained in the three cross-sectional planes $z_{1} / R=1.72, z_{3} / R=3.82, z_{5} / R=5.93$ cf. Fig. 1 , and compare the results at these positions with the measurements of ?.

We characterise the flow with two dimensionless numbers. The Reynolds number

$$
\operatorname{Re}=\frac{2 R V_{\mathrm{b}}}{\nu}
$$

and the swirl number

$$
\mathrm{S}=\frac{F_{\theta z}}{R F_{z z}}=\frac{\int_{0}^{R} \rho V_{\theta} V_{z} r \mathrm{~d} r}{R \int_{0}^{R} \rho V_{z}^{2} \mathrm{~d} r} .
$$

$\mathrm{S}$ expresses the ratio of the axial component of the angular momentum flux $\left(F_{\theta z}\right)$ and axial component of the momentum flux $\left(F_{z z}\right)$. A drawback of the definition of $\mathrm{S}$ is that it requires knowledge of the entire $V_{\theta}$ - and $V_{z}$-fields in a given cross section, which is typically not known for experiments. In addition, $\mathrm{S}$ cannot be determined a priori - it is a consequence of the flow physics.

Instead, the swirl number is in this case approximated by the design swirl number

$$
\mathrm{S} \approx \mathrm{S}_{\mathrm{d}}=\frac{V_{\theta, \mathrm{i}}}{V_{r, \mathrm{i}}} \frac{R}{2 h}
$$

where $V_{\theta, \mathrm{i}}$ and $V_{r, \mathrm{i}}$ are specified at the inlet and $h$ is the width of the inlet (?).

With the present boundary conditions and flow setting we have $\operatorname{Re}=1.0 \cdot 10^{5}$ and $\mathrm{S}_{\mathrm{d}}=0.22$. In the experiment by ? they had $\mathrm{S}_{\mathrm{d}}=0.23$ and their results were insensitive to a change in Reynolds number from $\operatorname{Re}=3.3 \cdot 10^{4}$ to $\operatorname{Re}=6.5 \cdot 10^{4}$. Here we use of the measurements at $\operatorname{Re}=6.5 \cdot 10^{4}$ to compare with the simulations.

Each simulation is run such that a non-dimensional time $T=t V_{\mathrm{b}} / L_{\mathrm{c}}$ of at least $T_{2}=30$ is covered. To avoid initial transients, we start averaging from $T_{1}=25$. We calculate the error associated with the mean velocity magnitude prediction from the expression (?)

$$
\epsilon_{\left\langle u_{i}\right\rangle}=\sqrt{\frac{2 \tau_{\mathrm{i}}}{T_{2}-T_{1}}} \frac{\sigma_{u_{i}}}{\left\langle u_{i}\right\rangle}
$$

where $\tau_{\mathrm{i}}$ is the integral time scale (minimum time between statistically independent samples), $\left\langle u_{i}\right\rangle, \sigma_{u_{i}}$ is the mean and standard deviation of the velocity component $u_{i}$. We estimate $\tau_{\mathrm{i}}=0.035$ from the autocorrelation of a monitor point in the $z_{0}$-plane at $(x, y, z)=(0.27 R, 0,0.55 R)$, for the $100 \%$ open port case. Using Eq. (27) we obtain the error $\epsilon_{\bar{u}}$ of $10 \%$ in the $100 \%$ open port case and $4.6 \%$ in the $50 \%$ open port case.

Notice that in the following figures showing velocity profiles (Fig. 2 and Fig. 6), the SPIV measurements are translated in the $x$-direction such that the vortex center (on average) coincides with $x=0$. The measured off-axis position of the vortex center is no larger than $0.05 R$ and it is due to ever-present inaccuracies of the experiment. The uncertainty $\epsilon_{\langle u\rangle}^{\prime}=\sigma_{u} /(\langle u\rangle \sqrt{N})$ associated with the measured 
average velocity component is of order $1.5 \%(2.0 \%)$ for the measurements with $100 \%(50 \%)$ open intake. In the formula, $N$ is the number of independent samples.

\section{1. $100 \%$ open intake}

In this section we consider the case of fully open intake. We compare the simulated and measured mean velocity profiles, consider the spatial evolution of the swirl number, and comment on the three-dimensional mean velocity field.

In Fig. 2 the mean axial and tangential velocity profiles are shown for three streamwise positions $z_{1}, z_{3}, z_{5}$, cf. Fig. 1 . The measured mean axial profiles are symmetric with a velocity deficit in the central region, resulting in a wake-like profile. Furthermore, it is observed that the axial velocity profiles decrease radially in the range $|x / R| \gtrsim 0.5$.

The measurements show plateaus with high axial velocity $\left(V_{z} / V_{\mathrm{b}} \approx 2.0\right)$, and a central velocity deficit where the axial velocity is reduced to $V_{z} / V_{\mathrm{b}}=0.16$, cf. Fig. $2 a$. It is seen that the DOEM and LDOEM are the only models capable of capturing qualitatively the velocity deficit, where the LDOEM exhibits the best quantitative agreement with the measurements. It should be noted that outside the vortex core region $(x / R \gtrsim 0.2)$ the four numerical models predict the same profile of $V_{z}$.

It is interesting to note that despite the disagreements between simulated and measured axial flow (except for the LDOEM model) there is a remarkable agreement in the predicted tangential velocity profile, as seen in Fig. $2 b$. The $V_{\theta}$-profile is well fitted by the Lamb-Oseen vortex profile: $V_{\theta}=\left(\Omega c^{2} / x\right) \cdot\left(1-\exp \left(-x^{2} / c^{2}\right)\right)$, shown in green (?). The Lamb-Oseen vortex is fitted to the LDOEM data using $\Omega$ and $c$ as fitting parameters that minimize the residual in a root-mean-square sense.

In the downstream cross sectional plane shown in Fig. $2 c$, the axial velocity profile retains its wake-like profile although it is widened by diffusion and at $z_{5}$ (Fig. 2e) the axial profile is reduced to a plug flow. Note from Figs. $2 c e$ that at these positions all numerical models resolve qualitatively the wake-like axial profile, although they overpredict in the velocity magnitude at $x / R \lesssim 0.4$. The measured and simulated tangential velocity profiles in these positions agree well and, as previously, they are fitted well by appropriate Lamb-Oseen vortex profiles. It is worth noting that the significant viscous decay of the vortex profile observed in Fig. 2 is not reflected in the downstream evolution of the swirl number (Eq. (25)). The streamwise evolution of $\mathrm{S}$ is plotted and it is observed that it evolves in a nonmonotonic fashion cf. Fig. 3. Specifically, $\mathrm{S}$ increases from $\mathrm{S}=0.14$ at $z_{1}$ to $\mathrm{S}=0.19$ at $z_{4}$. In attempting to elucidate the mechanism that gives rise to this behavior it is noted that $\mathrm{S}$ expresses the ratio of fluxes of angular $\left(F_{\theta z}\right)$ and axial $\left(F_{z z}\right)$ momentum in the axial direction. Thus, $\mathrm{S}$ increases when the decay of $F_{z z}$ is faster than the decay of $F_{\theta z}$. This is in fact the case as is shown in Fig. 4, where a relatively rapid decay of $F_{z z}$ is observed in the range $1.0<z / R<3.0$. In the figure, the momentum fluxes have been normalized with the axial momentum carried by a plug-flow $F_{z z}^{\prime}=\rho V_{b}^{2} \pi R^{2}$. 
(a)

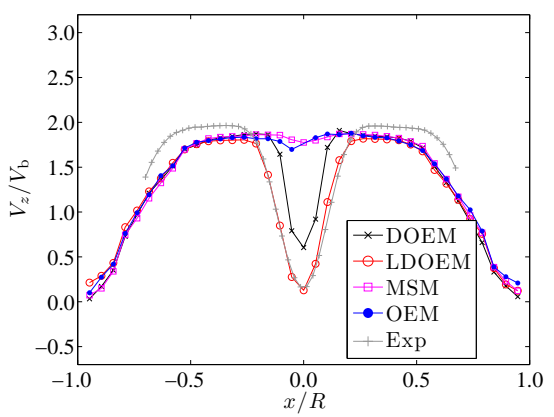

(c)

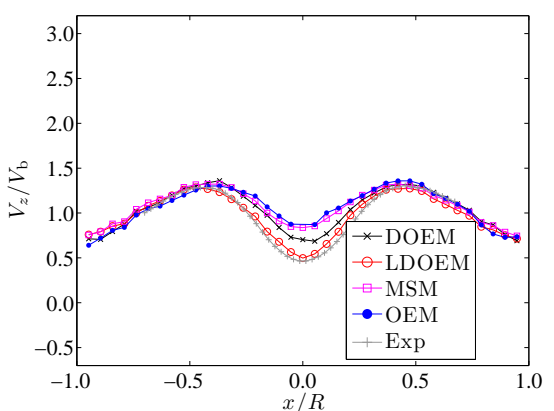

(e)

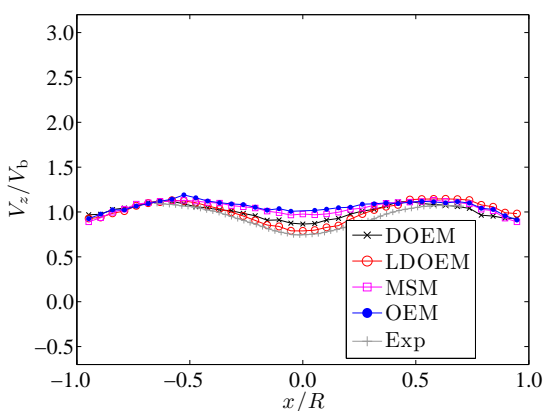

(b)

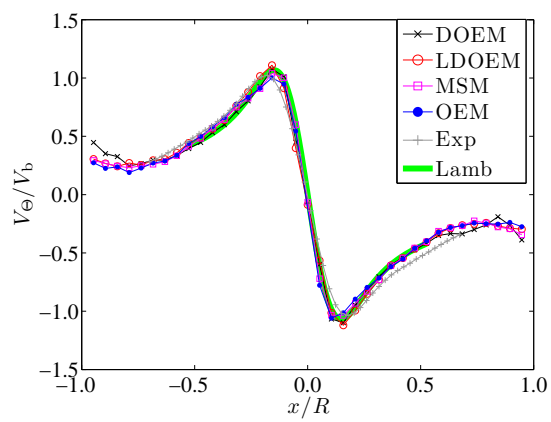

$(d)$

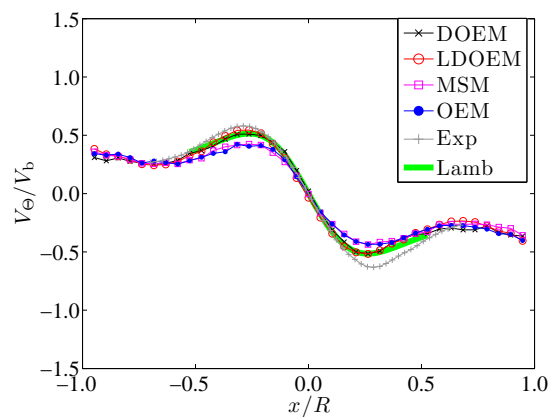

$(f)$

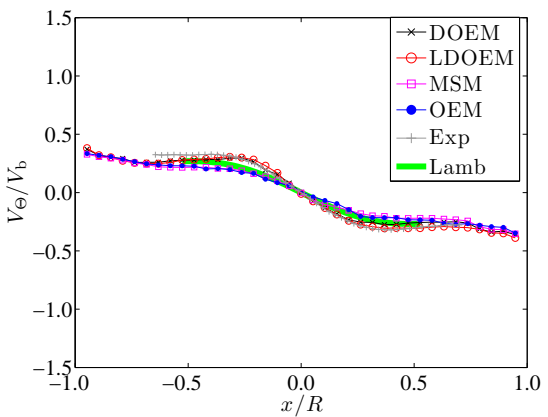

Fig. 2. Time averaged velocity profiles for the $100 \%$ open port case. The axial $(a, c, e)$ and tangential $(b, d, f)$ are obtained at the axial position $(a, b) z_{1} ;(c, d) z_{3} ;(e, f) z_{5}$. The abbreviations are "DOEM" (Dynamic one-equation model), 'OEM' (One-equation model), "LDOEM" (Localized Dynamic one-equation eddy-viscosity model), 'OEM' (One-equation model), "MSM" (Mixed-scale model), "Exp" (Measurements). The error associated with the measurements is of order $1.5 \%$.

We note that while a change in $F_{\theta z}$ presumably is governed by viscosity, the change in $F_{z z}$ is related to a change in the profile of the axial flow. Such changes can be caused by variations of the set-up's cross-section, or by the flow itself as e.g. vortex breakdown. In Fig. 4 we see that the decay of the axial flow profile, Figs. 2 ace, is accompanied by $F_{z z} / F_{z z}^{\prime} \rightarrow 1$, as expected. In positions further downstream we expect $F_{z z}$ to increase again due to the upstream effect of the reduced exhaust 
$\mathrm{z} 1 z_{1} \quad \mathrm{z} 2 z_{2} \quad \mathrm{z} 3 z_{3} \quad \mathrm{z} 4 z_{4} \mathrm{z} 5 z_{5} \quad \mathrm{z} 6 z_{6}$

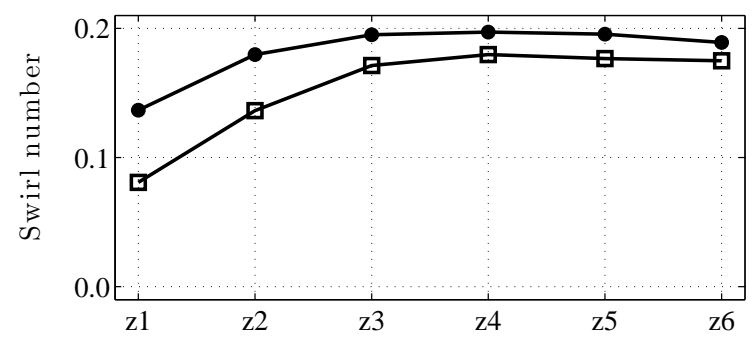

Fig. 3. The swirl number decays along the computational mesh for the case of $100 \%$ open intake (bullets) and case of $50 \%$ open intake (open boxes). The exact coordinate of the abscissa labels are given in the caption of Fig. 1.

$\mathrm{z} 1 z_{1} \quad \mathrm{z} 2 z_{2} \quad \mathrm{z} 3 z_{3} \quad \mathrm{z} 4 z_{4} \quad \mathrm{z} 5 z_{5} \quad \mathrm{z} 6 z_{6}$

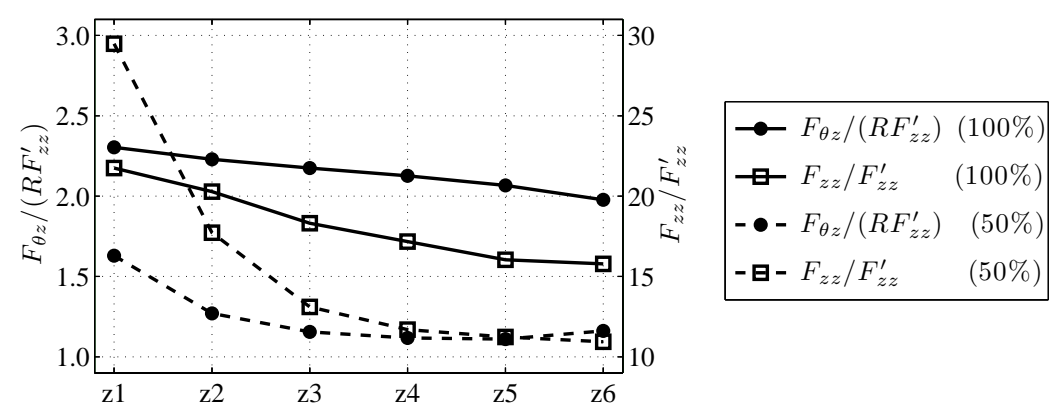

Fig. 4. Momentum flux as function of the downstream position. Notice that the overall decay of angular momentum flux in the axial direction $F_{\theta z}$ is slower than the decay of axial momentum flux $F_{z z}$, which leads to the observed increase in swirl number, cf. Fig. 3.

diameter.

Figure 5 gives an impression of the three-dimensional mean flow for the $100 \%$ open port case. Green and blue colors show streamlines that are released on two lines parallel with the $z$-axis in a meridional plane at the inlet $x= \pm 2.1 R$. As observed in the inset, the streamlines move inwards along an almost straight line, before winding around the axisymmetric vortex core. Notice also from both figures the braid of red streamlines at the beginning of the main cylinder. The streamlines are released inside the recirculation zone and they effectively show the toroidal recirculation zone through which the other streamlines flow. Notice from the side view how the streamlines rotate approximately one-half revolution as they travel downstream in the main cylinder. 


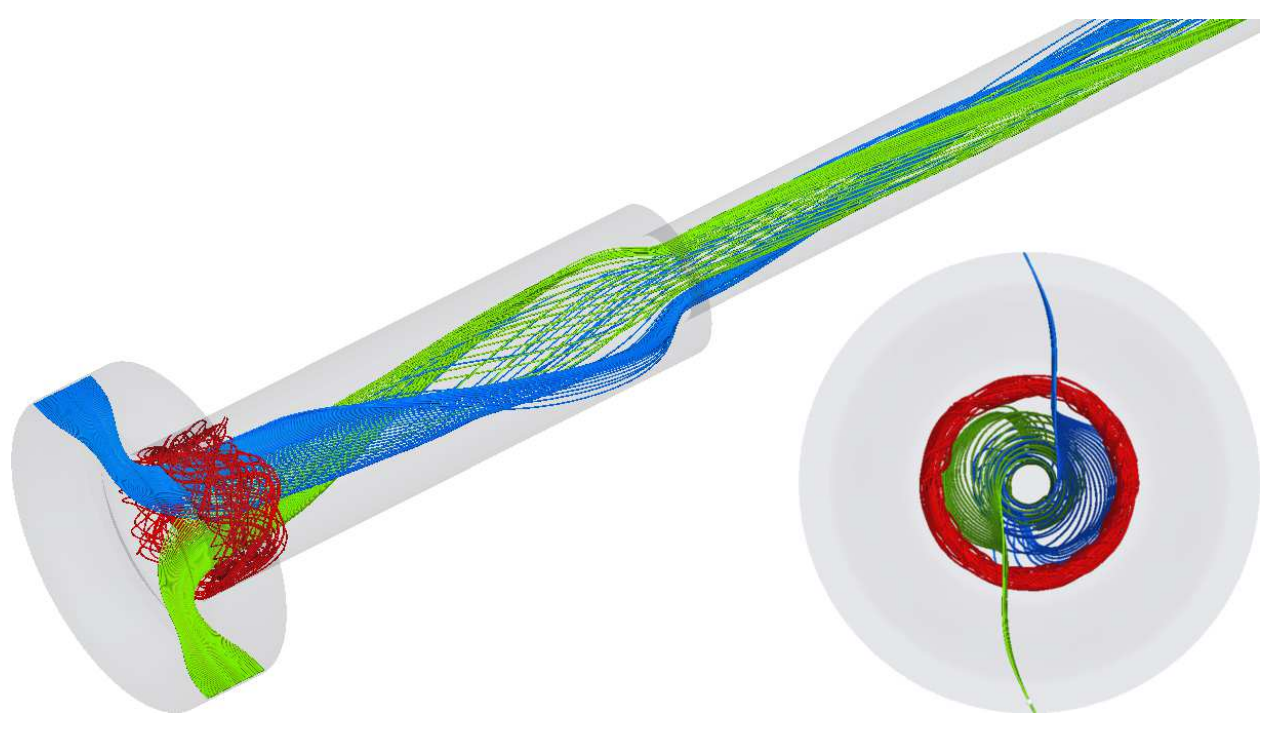

Fig. 5. Visualization of the three-dimensional flow by streamlines of the mean-field for the $100 \%$ open port case. Notice the braid of red streamlines that shows the recirculation region at the upstream end of the main cylinder and the large pitch of the flow in the main cylinder. The lower right inset is a bottom-view that shows the swirling streamlines.

\section{2. $50 \%$ open intake}

We now consider a situation analogous to the piston covering half the intake, i.e. the computational domain is as shown in Fig. 1. The flow is simulated using the LDOEM only since it was found to agree best with measurements in the $100 \%$ open intake case. The displaced piston presents a forward facing step to the flow, and flow separation now takes place at the sharp corner. From Fig. $6 a$ it is apparent that displacing the piston has a dramatic effect on the mean flow profiles. In contrast to the case of fully open intake (Fig. 2) the mean axial flow now assumes an overall jet-like and symmetric profile in the measurements. The LDOEM predicts a profile in reasonable agreement with the measured profile. Notice that the axial flow is reversed in the region $|x| / R \gtrsim 0.8$, which indicates that the recirculation bubble extends further downstream compared to the $100 \%$ open intake case. We will elaborate further on this observation in section 4.3.

The simulated and measured mean tangential velocity profiles shown in Fig. 6b, display a poor agreement. The simulated flow has the overall tangential profile of a Lamb-Oseen vortex as shown by the green curve. In contrast, the measured profile is monotonically decreasing and it does not have a profile of a "standard" vortex. Upon comparison of the $V_{\theta}$-profiles of Fig. $2 b$ and Fig. $6 b$ it is interesting to note the difference in slope of the simulated tangential velocity profile near $x=0$. For the $100 \%$ open intake case, the slope of a linear fitting curve is -10.0 and for the $50 \%$ open intake case the slope is -5.3 , which shows that the maximum axial vorticity 
(a)

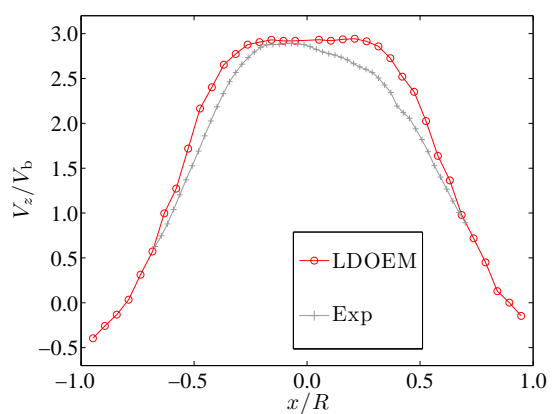

(c)

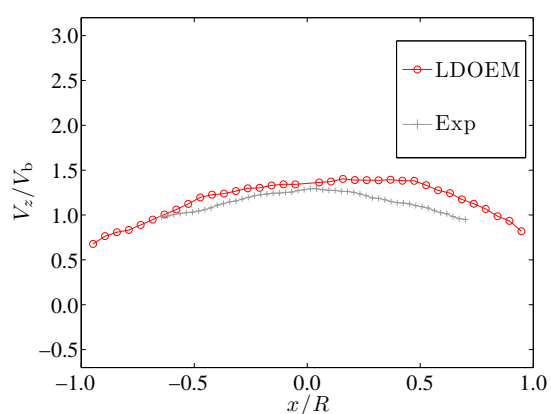

$(e)$

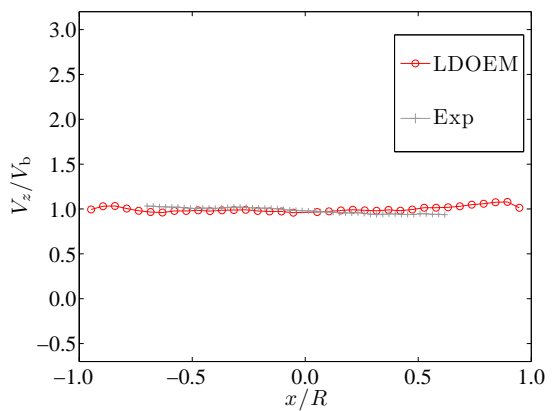

(b)

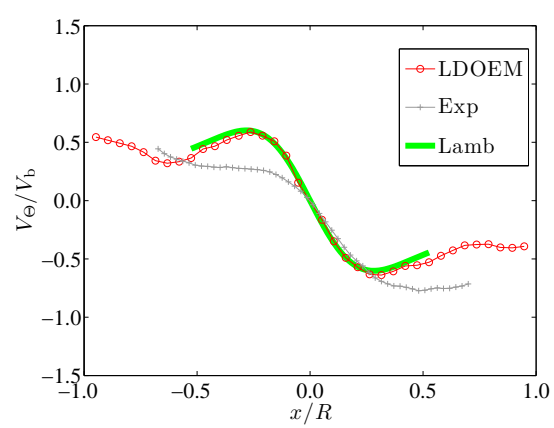

$(d)$

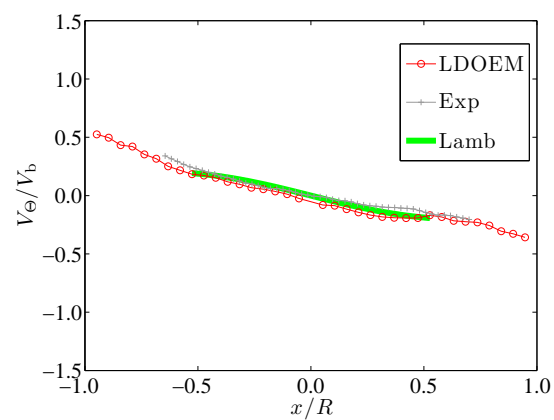

$(f)$

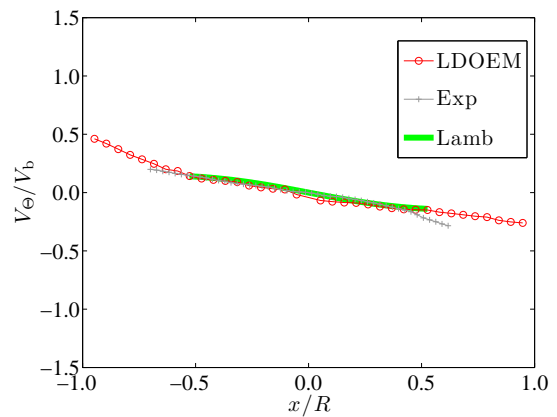

Fig. $6.50 \%$ open intake case. $(a, c, e)$ show the time averaged axial velocity profiles and $(b, d, f)$ show the time averaged tangential velocity profiles. The axial positions are $(a, b) z_{1} ;(c, d) z_{3} ;(e, f)$ $z_{5}$. The error associated with the measurements is of order $2.0 \%$.

$\omega_{z}=(1 / r) \partial\left(r V_{\theta}\right) / \partial r$ is reduced by $47 \%$ in the case of $50 \%$ open intake.

In the downstream position $z_{3}$, the axial flow persists having a jet-like profile cf. Fig. $6 c$, with a good qualitative agreement between the measured and simulated flows. The simulated profiles overestimate the measured profile by $10 \%$ at $x=0$. Notice from the tangential velocity profiles shown in Fig. $6 d$ that it has the signature of solid-body rotation where in fact the thin wall boundary layer is not resolved by the shown data acquisition points. We estimate the boundary layer thickness from that of the flow past a flat plate $\delta / R \approx 0.37 /(\mathrm{Re})^{-1 / 5}(?)$. We obtain $\delta / R=0.03$ 
which is closer to the wall than captured by the velocity monitor points shown in Fig. 6.

In the downstream position $z_{5}$ the axial velocity profile has taken the form of a plug-flow with solid-body rotation, cf. Fig. 6 ef. Only a very narrow boundary layer exists near the cylinder wall. A similar profile was observed in the case of $100 \%$ open intake, cf. Fig. $2 e$. The tangential profile remains solid-body like although the overall swirl decreases downstream due to viscosity. These observations are supported by the streamwise evolutions of the momentum fluxes, cf. Fig. 4 . Here it is seen that the jet-like profile yields a $80 \%$ larger $F_{z z}$ compared to the case of $100 \%$ open intake. Again we observe a downstream decrease of $F_{z z}$. Notice that $F_{\theta z}$ is only slightly reduced, $5 \%$ at $z_{1}$, compared with the $100 \%$ open intake case. The succeeding decay of $F_{\theta z}$ is more rapid. We explain this observation with the stronger shear that yields an increased viscous decay of the angular momentum flux. In Fig. 3 we show the evolution of $\mathrm{S}$ as function of the downstream position (open boxes) for the present case and observe that, as expected, $\mathrm{S}$ assumes a smaller value than for the case of $100 \%$ open intake.

The qualitative appearance of the streamlines (not shown) is similar to the $100 \%$ open intake case (Fig. 5), i.e., a toroidal recirculation zone (of the average flow field) is located at the cylinder wall immediately downstream of the inlet section. It is therefore worth noting that one cannot characterize the profile of the axial velocity profile from visual inspection of the mean velocity-based streamlines alone.

\subsection{Flow structures}

Figure 7 shows the time-averaged velocity magnitude for the case of $100 \%$ open intake (Fig. $7 a$ ) and for the case of $50 \%$ open intake (Fig. $7 c$ ). Notice here that the most significant difference in flow is observed immediately downstream of the inlet section. In both cases, the recirculation zone (on average) is located downstream of the corner where the flow turns from a predominantly radial to a predominantly axial direction. We estimate the streamwise extend of the separation zone from the sign of the axial velocity close to the cylinder wall and find that the streamwise length of the separation zone is $0.72 R$ for the $100 \%$ open intake case and $1.2 R$ for the $50 \%$ open intake case. The reason why the separation zone extends further downstream in the $50 \%$ open intake case is the strong jet that is formed as flow enters the cylinder through the reduced intake. The qualitatively different nature of the axial flow profiles investigated in Fig. 2 and Fig. 6 is clearly recognizable in the medridional mean fields. Notice again that the velocity magnitude fields in the downstream third of the cylinder length are almost identical, as shown by the axial velocity profiles of Fig. $2 e$ and Fig. $6 e$. A qualitative difference between the two mean velocity magnitude fields is the presence of two recirculation zones at the protruding piston. These are shown by selected streamlines in Fig. $7 e$. The panel shows a zoom of the region marked by a dotted line in Fig. $7 c$. We note that the shown streamlines are in fact three-dimensional streamlines projected onto the 


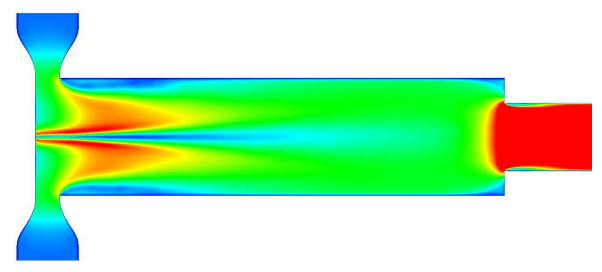

(a)

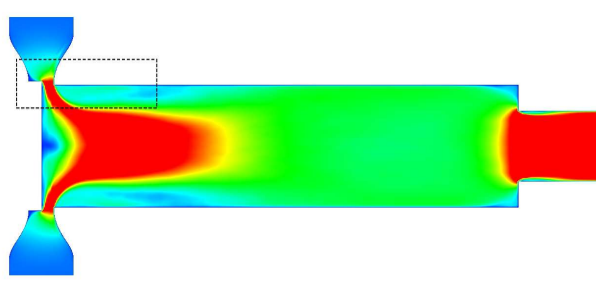

(c)

(e)

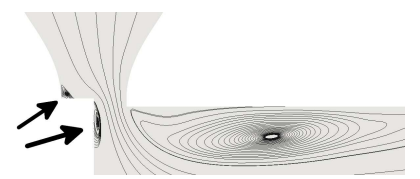

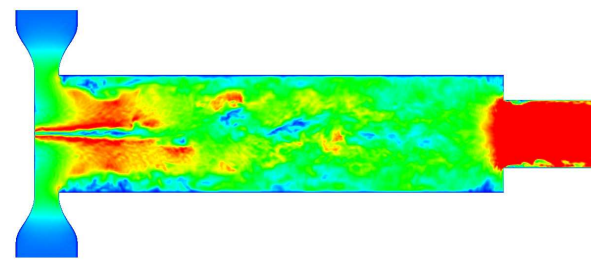

(b)

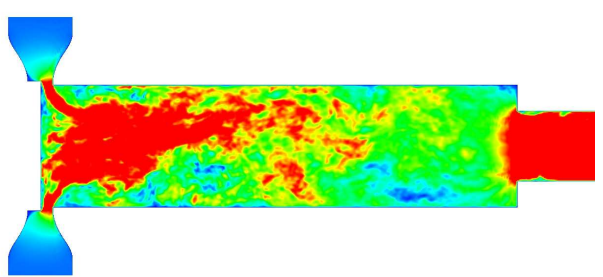

$(d)$

112200 NNon-dimensional velocity magnitude $\mathrm{N}$

Fig. 7. Meridional planes showing the velocity magnitude fields. $(a, c)$ show the mean velocity magnitude for the cases of $100 \%$ and $50 \%$ open intake, respectively, and $(b, d)$ show instantaneous velocity magnitudes for the same cases. In panel $(e)$, selected stream lines show recirculation zones, two of which are shown with arrows, in the dotted region of panel $(c)$.

meridional plane. We observe a small recirculation zone before the step, marked by an arrow, and a larger recirculation zone on the piston surface. No recirculation zones were observed in the mean velocity magnitude field for the case of $100 \%$ open intake.

In Fig. $7 b d$ we show typical examples of the instantaneous velocity magnitude field in the meridional plane, where the highly unsteady nature of the flow is evident, particularly in the $50 \%$ open intake case. In the case of $100 \%$ open intake we observe a symmetric velocity field, reminiscent of the mean field, whereas in the $50 \%$ open intake case pronounced bursts of high-speed regions are seen. Presumably, these bursts appear due to interactions of shear layers created at the strong jets, which lead to large-scale disturbances of the flow.

Fig. 8 shows the time averaged vorticity magnitude in the meridional planes. We observe in the case of $100 \%$ open intake (Fig. $8 a$ ), that a region of high vorticity 
(a)

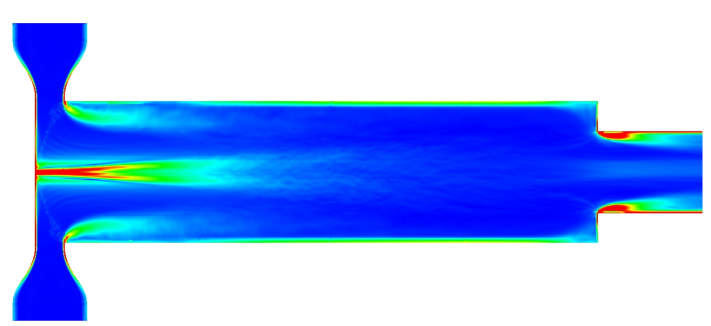

$00101020202525 \mathrm{~N}$

(b)

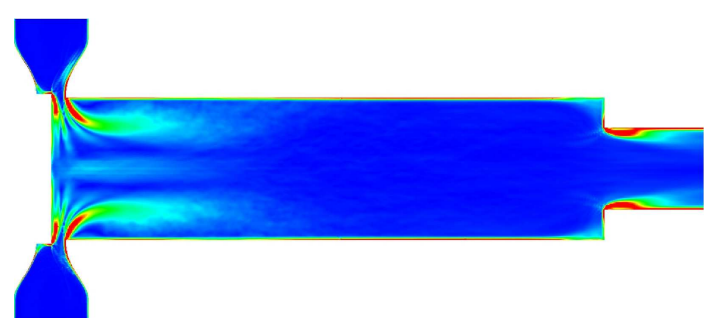

0

10

20

Non-dimensional vorticity magnitude $\mathrm{N}$

Fig. 8. Meridional views showing the time averaged vorticity magnitude for the case of $100 \%$ open intake $(a)$ and the case of $50 \%$ open intake $(b)$.

exist in the center of the cylinder effectively showing the compact vortex core. A moderate production of circulation is likewise observed at the sharp corner marked by an arrow. For the case of $50 \%$ open intake, (Fig. $8 b$ ) the vortex core is weaker, as discussed in Section 4.2, whereas a pronounced production of vorticity now takes place as the flow enters the cylinder.

\section{Discussion and conclusion}

High Reynolds number swirling flows are generally hard to predict numericallyin particular RANS models over-predict the decay of swirl. In this contribution, we simulate the scavenging and swirling flow in the static geometry of a simplified model of a large diesel engine using LES with four different turbulence models. Upon comparison with experimental results, obtained with stereo particle image velocimetry it is found that the both the "localized dynamic one-equation model" and the "dynamic one-equation model" (?????) yield qualitatively and quantitatively 
good predictions of the mean axial and tangential velocity profiles. In contrast, the two alternative models, the "one-equation model" (?) and the "mixed-scale model" (?) both fail to predict qualitatively the correct profile of the axial flow profiles.

We show that the position of the piston has a dramatic effect on the measured and simulated flow fields: displacing the piston such that the intake is covered by $50 \%$ (which corresponds to a $50 \%$ blockage of the scavenge ports of the engine) changes the time-averaged axial velocity profiles from a wake-like shape to a jet-like shape, and also diminishes the strength of the in-cylinder vortex. The partial intake closure also has more local consequences for the average velocity magnitude fields in the formation of two recirculation zones up- and downstream of the sharp corner. It is important to note that despite the difference in Reynolds number between the experiments by ?, good agreement between numerical and experimental results is observed.

We believe that our results carry two important messages. Firstly, the confined swirling flow can be predicted with a reasonable accuracy with LES using the dynamic one equation eddy model. Secondly, the piston position has a dominating influence on the in-cylinder flow, creating a qualitatively change in the flow topology.

\section{Acknowledgements}

We thank Valery L. Okulov and Dalibor Cavar for invaluable discussions and inputs. The research has been supported by the Danish Agency for Science Technology and Innovation (Grants No. 08-034075 and 09-070608), MAN Diesel \& Turbo, and the Danish Centre for Maritime Technology. We are grateful to ETH Zürich for providing computational resources. 